\title{
THE INVARIANT INTEGRAL AND THE INVERSE PROBLEM IN THE CALCULUS OF VARIATIONS*
}

\author{
BY \\ THOMAS H. RAWLES $\dagger$

\section{INTRODUCTION}

1. The simplest problem in the calculus of variations is the determination of that curve, $y=Y(x)$, joining two points, $P_{1}$ and $P_{2}$, in the $x y$ plane along which the definite integral

$$
J=\int_{x_{1}}^{x_{2}} f\left(x, y(x), y^{\prime}(x)\right) d x
$$

must be taken in order that the value of the integral may be an extremum. The differential equation which defines the desired curve is of the second order and its general solution is a two-parameter family of curves known as the extremals of the problem. By the successive substitution of the coördinates of the end points, $P_{1}$ and $P_{2}$, in the equation of the extremals we obtain two equations for the determination of the values of the parameters in terms of these coördinates.

2. The inverse problem is, as its name suggests, that of determining the form of the integrand function which has as its extremals a given twoparameter family of curves.

3. Besides the simplest problem as we have stated it there is the problem of determining the extremals of an integral,

$$
J=\int f\left(x, y_{1}(x), y_{2}(x), \cdots, y_{n}(x), y_{1}^{\prime}(x), y_{2}^{\prime}(x), \cdots, y_{n}^{\prime}(x)\right) d x,
$$

whose integrand function contains $n$ dependent variables and their derivatives. The extremal in this case is a curve in a hyperspace of $n+1$ dimensions and is defined by the intersection of $n$ hypersurfaces. The problem is of order $2 n$ and the constants are determined by successively substituting the coördinates of the two end points in the $n$ equations which define the extremals.

* Presented to the Society, December 28, 1927; received by the editors June 24, 1927.

† Sterling Fellow in Mathematics, Yale University, 1926-1927. 
Corresponding to this there is of course an inverse problem in which the extremals are given by a family of curves involving $2 n$ parameters in a hyperspace of $n+1$ dimensions.

4. The inverse problem in the calculus of variations was first discussed by Darboux* who was led to its study in connection with his researches in the theory of surfaces. He was able to apply his results to certain problems which had been previously investigated by Beltrami and Dini. He also solved the problem of finding the form of the integrand function which has families of straight lines as its extremals.

5. The first application of Darboux's ideas was that of Hamel, $†$ who investigated the geometries in which it is assumed among other axioms that the shortest distance between two points is measured along a straight line joining them. He first developed the most general form of a line element of such a geometry in a plane. This portion of Hamel's paper contains nothing but what is a direct consequence of Darboux's work. He found in addition the form of the integrand function which has as its extremals straight lines in space.

6. Employing methods similar to those of Hamel, Stromquist $\ddagger$ was able to obtain the most general form of line element of a geometry in which the shortest distance between two points is measured along the segment of a circle which joins them and has its center on the boundary of the half plane. He then introduced the restriction that the transversals are perpendicular to the extremals and showed that this leads to a unique form of line element.

7. Miles§ obtained the integrand functions which have as extremals certain two-parameter families of conics. This investigation as well as those of Hamel and Stromquist is a direct application of Darboux's methods.

8. By using as the independent variable the angle between the tangent and the positive direction of the $x$ axis Bliss $\|$ was able to discuss the problem without restricting himself to curves whose tangents do not become parallel to the axis of the dependent variable. In order to determine the integrand function uniquely he assigned a particular family of transversals to any one-parameter family of extremals which form a field.

9. Since the methods developed by Darboux and Bliss give an infinite number of solutions a further condition must be imposed in order to give

- Darboux, Theorie des Surfaces, vol. III, $\$ 8604,605$.

$\dagger$ Hamel, Mathematische Annalen, vol. 57 (1903), p. 231; also his dissertation, Göttingen, 1901.

† Stromquist, these Transactions, vol. 7 (1906), p. 181.

\& Miles, Ametican Mathematical Monthly, vol. 20 (1913), p. 117.

|| Bliss, Annals of Mathematics, (2), vol. 9 (1907), p. 127. 
uniqueness to the result. The condition which naturally suggests itself is one which relates the slope of the extremals to that of an associated family of curves known as the transversals.* When such a condition is imposed the determination of the integrand function becomes unique except for a constant factor which has no significance as far as the first variation is concerned.

10. The objects of this paper are as follows:

I. To determine the general form which the integrand function must have in order that there may exist a prescribed relation between the slope of the extremal and that of the normal to the transversal.

II. Having found the general form of the integrand function, to determine the particular function which has as its extremals a given family of curves.

The cases of one and $n$ dependent variables will be given separate discussion with the same ends in view in order to bring out certain conditions which must be satisfied when more than one dependent variable is present.

The problem will be attacked by means of the Hilbert invariant integral.

\section{The CASE OF ONE DEPENDENT VARIABLE}

1. The field of extremals. A one-parameter family of extremals, $y=Y(x$, $a$ ), is said to form a field $\dagger$ when in a region which we may define by $a_{1} \leqq a \leqq a_{2}$, and $x_{1} \leqq x \leqq x_{2}$, no two curves of the family have a point in common. This implies of course that for any pair of values of $x$ and $y$ in such a region the equation $y=Y(x, a)$ has one and but one root in $a$. Therefore we may define a function $a=A(x, y)$, known as the inverse function of the field. Since the extremals of the field have continuous first derivatives there is a definite slope associated with each point of the field which we shall denote by

$$
p(x, y)=Y_{x}(x, A(x, y)),
$$

calling it the slope function of the field.

An important theorem on the existence of a field is the following. Suppose that a family of extremals, $y=Y(x, a)$, contains the curve $C_{0}$ for $a=a_{0}$, and that the functions $Y$ and $Y_{x}$ are of class $C^{\prime}$ in the region which satisfies the inequalities $X_{1} \leqq x \leqq X_{2}$, and $\left|a-a_{0}\right|<d$; and also that $X_{1}<x_{1}$ $<x_{2}<X_{2}$. Then if $Y_{a}(x, a) \neq 0$ in the open interval $\left(x_{1}, x_{2}\right)$, a quantity $k$ may be chosen so small that the family of curves $y=Y(x, a)$ forms a field about the curve $C_{0}$ when $x_{1} \leqq x \leqq x_{2}$, and $\left|a-a_{0}\right|<k$.

* Bliss, loc. cit., p. 134; Stromquist, loc. cit., p. 180.

$\dagger$ Bolza, Vorlesungen über Variationsrechnung, Berlin, 1909, p. 96.

$\ddagger$ Bolza, Vorlesungen, p. 100. 
2. The invariant integral. It was shown by Hilbert $\dagger$ that the integral

$$
J^{*}=\int_{P_{0}}^{P} f(x, y, p(x, y))+\left(y^{\prime}-p(x, y)\right) f_{y^{\prime}}(x, y, p(x, y)) d x,
$$

taken along any curve lying wholly within the field, is independent of the path of integration and depends only upon the coördinates of the end points. If, therefore, $J^{*}\left(P_{0}, P\right)$ represents the integral taken from a point $P_{0}$ to any other point in the field it will define a function,

$$
J^{*}\left(P_{0}, P\right)=w(x, y) .
$$

It is known further $\ddagger$ that the derivatives of this function are

(4)

$$
\begin{aligned}
\frac{\partial w}{\partial x} & =f(x, y, p(x, y))-p(x, y) f_{y^{\prime}}(x, y, p(x, y)), \\
\frac{\partial w}{\partial y} & \left.=f_{y^{\prime}}(x, y, p x, y)\right) .
\end{aligned}
$$

The family of curves $w=$ constant are known as the transversals of the field. They are of class $C^{\prime \prime}$, and along any one of them the invariant integral has a constant value. We shall call $w$ the transversal function of the field.

If from equations (4) we eliminate $p$, a partial differential equation in $w$ is obtained. Then, if there can be found in any way a solution of this equation containing a non-linear constant, $\alpha$, the extremals are given by

$$
\frac{\partial w(x, y, \alpha)}{\partial \alpha}=c
$$

where $c$ is the second arbitrary constant which the solution of the Euler equation must contain.

3. Having in mind these properties of the field, and of the transversal function associated with it, we shall now seek the form which the integrand function must assume in order that a given relation between the slope of the extremal and that of the normal to the transversal shall be satisfied.

Let us suppose that the extremals are given by equations (5), and that the slope of the transversals of the field is related to that of the extremals by a given function of the slope of the extremals and of the coördinates of the point,

$$
w_{y}=m(x, y, p) w_{x}
$$

† Bolza, Vorlesungen, p. 106.

† Bolza, Vorlesungen, p. 129. 
We shall define as a transversality any relation such as (6) in which the function $m$ is of the class $C^{\prime}$, excepting only the function defined by $1+m p=0$.

Now, if we differentiate (5) with respect to $x$ the result is

$$
w_{\alpha x}+w_{\alpha y} y^{\prime}=w_{\alpha x}+w_{\alpha y} p=0 .
$$

This serves to define $p$, the slope function of the field.

In order to proceed with the discussion of the problem we shall fix our attention on that portion of the field for which $1+m p \neq 0$ and $w_{x} \neq 0$. Differentiating (6) with respect to $\alpha$ we obtain

This becomes

$$
\frac{w_{y \alpha}}{w_{x}}-\frac{w_{y} w_{x \alpha}}{w_{x}^{2}}=m_{\alpha} .
$$

$$
-\frac{1}{p} \frac{w_{x \alpha}}{w_{x}}-m \frac{w_{x \alpha}}{w_{x}}=m_{\alpha}
$$

when we replace $w_{\nu}$ and $w_{y \alpha}$ by their values which may be obtained from (6) and (7) respectively.

Solving this last equation for $w_{x \alpha} / w_{x}$ we find

$$
\frac{w_{x \alpha}}{w_{x}}=-\frac{m_{\alpha} p}{1+m p}
$$

or, after adding and subtracting the term $m p_{\alpha} /(1+m p)$,

$$
\frac{w_{x \alpha}}{w_{x}}=-\frac{\partial}{\partial \alpha} \log (1+m p)+\frac{m p_{\alpha}}{1+m p} .
$$

Upon integrating this we now obtain

where

$$
w_{x}=g(x, y)\left(\frac{1}{1+m p}\right) e^{\ominus},
$$

$$
\Theta=\int \frac{m d p}{1+m p},
$$

and $g(x, y)$ is an arbitrary function of its arguments.

By means of equations (6) and (8) we at once arrive at

$$
w_{y}=g(x, y)\left(\frac{m}{1+m p}\right) e^{\ominus} .
$$

We now substitute these expressions for $w_{x}$ and $w_{y}$ in (4) and find

$$
f(x, y, p)=w_{x}+p w_{y}=g(x, y) e^{\theta} .
$$


This result was first given by Stromquist* in 1907. The method by which we have derived it, employing as it does the invariant integral, is quite different from that which he used.

4. Having found the general form of the integrand function for a specified transversality, we now suppose that, in addition, a two-parameter family of curves, $\phi(x, y, a, c)=0$, is given and we seek to determine the particular integrand function for which they are extremals.

Assuming that in a region in the $x y$ plane, and for certain values of $a$ and $c, \phi_{c}$ is not equal to zero, it follows that when $a$ is fixed and $c$ is given nearby values the family of extremals thus obtained forms a field. This is a consequence of the theorem stated in the first paragraph of this section.

Moreover, we can solve for $c$, finding

$$
C(x, y, a)=c .
$$

Then we may differentiate with respect to $x$ and obtain

$$
p=-\frac{C_{x}}{C_{y}}
$$

where $p$ is the slope function of the field.

The existence of a field implies the existence of a family of transversals, $w(x, y, \alpha)=$ constant, from which we can derive the extremals by differentiation with respect to $\alpha$. The extremals would be given by

$$
w_{\alpha}(x, y, \alpha)=\gamma .
$$

Now, when we differentiate this with respect to $x$, we find

$$
p=-\frac{w_{\alpha x}}{w_{\alpha y}},
$$

where $p$ is again the slope function of the field. Since the expression obtained from (15) is identical with that obtained from (13) it follows that $a$ must be some function of $\alpha$.

Since $w$ is of class $C^{\prime \prime}$ we must have

Therefore, it follows that

$$
w_{x y}=w_{y x} .
$$

$$
\frac{\partial}{\partial y}\left(\frac{g e^{\Theta}}{1+m p}\right)=\frac{\partial}{\partial x}\left(\frac{m g e^{\Theta}}{1+m p}\right) .
$$

* Stromquist, Annals of Mathematics, (2), vol. 9 (1907), p. 57. 
This may be written

$$
\begin{aligned}
\frac{m g_{x}}{g}-\frac{g_{y}}{g} & =\frac{\partial}{\partial y}(\Theta-\log (1+m p))-m_{x}-m \frac{\partial}{\partial x}(\Theta-\log (1+m p)) \\
& =\Phi(x, y, \alpha) .
\end{aligned}
$$

This is a partial differential equation for the determination of $g(x, y)$. To obtain a solution we make use of the fact that $g$ is independent of $\alpha$ while it can be shown that $m$ must contain $\alpha$. For, if $m$ were independent of $\alpha$ we would have

$$
w_{y \alpha}-m w_{x \alpha}=0,
$$

from (6). But by (15)

$$
w_{x \alpha}+p w_{y \alpha}=0 \text {. }
$$

If the determinant of the system formed by these two equations is to vanish we must have

$$
1+m p=0 \text {, }
$$

which is contrary to an assumption which we have made.

Differentiating (16) with respect to $\alpha$, or, what amounts to the same thing, with respect to $a$, and dividing by $-m_{\alpha}$ we obtain

$$
\frac{g_{x}}{g}=\frac{1}{m_{\alpha}} \Phi_{\alpha} .
$$

Substituting this result in (16) we find at once

$$
\frac{g_{y}}{g}=\frac{m}{m_{\alpha}} \Phi_{\alpha}-\Phi .
$$

Knowing the partial derivatives of $\log g$ we can easily find $g$ by integration.

It is not difficult to show that the solutions thus obtained are unique except for a constant factor, which, as we have already remarked, has no significance as far as the first variation is concerned. For, if there were two solutions $g$ and $g^{\prime}$, we would have

$$
\left(\frac{g_{y}}{g}-\frac{g_{y}^{\prime}}{g}\right)-m\left(\frac{g_{y}}{g}-\frac{g_{x}^{\prime}}{g}\right)=0,
$$

which may be written

$$
\frac{\partial}{\partial y} \log \frac{g}{g^{\prime}}-m \frac{\partial}{\partial x} \log \frac{g}{g^{\prime}}=0 .
$$


Since $g$ and $g^{\prime}$ are independent of $\alpha$, and $m$ is not, this is impossible unless $g / g^{\prime}$ is a constant.

Example I. Let us assume that the prescribed transversality is that the transversals shall intersect the extremals orthogonally. If this is the case then $m=p$ and

$$
\Theta=\int \frac{m d p}{1+m p}=\int \frac{p d p}{1+p^{2}}=\frac{1}{2} \log \left(1+p^{2}\right) .
$$

The integrand function, therefore, is of the form

$$
f=g(x, y)\left(1+p^{2}\right)^{1 / 2} .
$$

Further, let us suppose that the prescribed family of curves which are to be the extremals of the problem is the family of parabolas given by

$$
y=a+\frac{(x+c)^{2}}{4 a} .
$$

First, we notice that we can solve this equation for $c$. After differentiating with respect to $x$ we calculate $p$, and then $\Phi$ by substitution in (16). The result is

$$
\begin{aligned}
p= & \left(\frac{y-a}{a}\right)^{1 / 2}, \\
\Phi= & -\frac{1}{2} \frac{\partial}{\partial y} \log \left(1+p^{2}\right)-p_{x}+\frac{p}{2} \frac{\partial}{\partial x} \log \left(1+p^{2}\right) \\
= & -\frac{1}{2} \frac{\partial}{\partial y}[\log y-\log a]-\frac{\partial}{\partial x}\left(\frac{y-a}{a}\right)^{1 / 2} \\
& +\frac{1}{2}\left(\frac{y-a}{a}\right)^{1 / 2} \frac{\partial}{\partial x} \log \frac{y}{a}=-\frac{1}{2 y} .
\end{aligned}
$$

On substituting these results in (17) and (18) we see that

$$
\frac{g_{x}}{g}=0, \quad \frac{g_{y}}{g}=\frac{1}{2 y} .
$$

Hence $g=$ constant $y^{1 / 2}$.

On the other hand we might wish to solve for $a$. In this case we would obtain

$$
a=\frac{y \pm\left(y^{2}-(x+c)^{2}\right)^{1 / 2}}{2} .
$$


Differentiating this with respect to $x$ we then find

$$
p=\frac{x+c}{x \pm\left[y-(x+c)^{2}\right]^{1 / 2}} .
$$

When this result is substituted in $\left(16^{\prime}\right)$ that equation becomes

$$
\begin{aligned}
\Phi= & -\frac{1}{2}\left(\frac{1}{y}-( \pm) \frac{1}{\left(y^{2}-(x+c)^{2}\right)^{1 / 2}}\right) \\
& -\frac{\left(y \pm\left(y^{2}-(x+c)^{2}\right)^{1 / 2}\right)\left(y^{2}-(x+c)^{2}\right)^{1 / 2} \pm \frac{1}{2}(x+c)^{2}}{\left(y \pm\left(y^{2}-(x+c)^{2}\right)^{1 / 2}\right)^{2}\left(y^{2}-(x+c)^{2}\right)^{1 / 2}} \\
= & -\frac{1}{2 y}-\frac{-\frac{1}{2}( \pm)\left(y \pm\left(y^{2}-(x+c)^{2}\right)^{1 / 2} \pm\left(y^{2}-\frac{1}{2}(x+c)^{2}\right)+y\left(y^{2}-(x+c)^{2}\right)^{1 / 2}\right.}{\left(y^{2}-(x+c)^{2}\right)^{1 / 2}\left(y \pm\left(y^{2}-(x+c)^{2}\right)^{1 / 2}\right)^{2}} \\
= & -\frac{1}{2 y}
\end{aligned}
$$

as before.

5. While every two-parameter family of curves may be regarded as the extremals of a problem in the calculus of variations it does not follow that any family may serve as the extremals of a problem with a prescribed transversality. The necessary conditions to be satisfied in order that it shall do so are easily obtained.

Since the left member of (17) is independent of $a$ the right member must also be independent. From this it follows that

$$
\Phi_{a a}-\frac{m_{a a}}{m_{a}} \Phi_{a}=0
$$

When the right member of (17) is independent of $a$ the right member of (18) is likewise. For differentiating the right member of (18) we have

$$
\frac{m}{m_{a}}\left(\Phi_{a a}-\frac{m_{a a}}{m_{a}} \Phi_{a}\right)-\Phi_{a}+\frac{m_{a}}{m_{a}} \Phi_{a}=\frac{m}{m_{a}}\left(\Phi_{a a}-\frac{m_{a a}}{m_{a}} \Phi_{a}\right) .
$$

This vanishes on account of (19).

A second condition to be satisfied is that equations (17) and (18) shall actually define a function. This requires that

$$
\frac{\partial}{\partial x}\left(\Phi+\frac{m}{m_{a}} \Phi_{a}\right)=\frac{\partial}{\partial y}\left(\frac{1}{m_{a}} \Phi_{a}\right)
$$

These conditions are also sufficient. For when they are satisfied there exists a function, $w$, whose derivatives are given by 
and

$$
w_{x}=\frac{g e^{\Theta}}{1+m p},
$$

$$
w_{y}=\frac{m g e^{\theta}}{1+m p} .
$$

Since these derivatives satisfy (16), which may be written

$$
\frac{\partial}{\partial y}\left(f-p f_{p}\right)=\frac{\partial}{\partial x} f_{p},
$$

and since any function $p$ which does satisfy this equation is a slope function of a field of extremals, our given curves are actually the extremals of the integrand function which we have found.*

Example II. A two-parameter family of curves which does not satisfy (19) for the orthogonal transversality, $m=p$, is $y=a(x-c)^{2}$. Here

Condition (19) becomes

$$
p=2(a y)^{1 / 2}, \quad \Phi=\frac{2}{(1 / a)+4 y} .
$$

$$
\frac{p_{a a}}{p_{a}} \Phi_{a}-\Phi_{a a}=-\frac{1}{2 a} \frac{2}{(1+4 a y)^{2}}+\frac{16 y}{(1+4 a y)^{3}} \not \equiv 0 .
$$

Example III. A two-parameter family in which (19) is satisfied but (20) is not, is the following:

$$
\begin{aligned}
& y-\int_{0}^{x} \tan \left(x^{2}-a\right) d x=c, \\
& p=\tan \left(x^{2}-a\right), \quad \Phi=-2 x .
\end{aligned}
$$

Condition (20) becomes

which is not satisfied.

$$
\frac{\partial}{\partial x}(-2 x)=\frac{\partial}{\partial y}(0)
$$

II. The CASE of $\boldsymbol{n}$ DEPENDENT VARIABLES

1. Analogous to the transversal lines in the case of one dependent variable and to the transversal surfaces in the case of two, we have here to deal with a family of transversal hypersurfaces in a space of $n+1$ dimensions. These we shall indicate by $w\left(x, y_{1}, y_{2}, \cdots, y_{n}, \alpha_{1}, \alpha_{2}, \cdots, \alpha_{n}\right)=c$, or, more briefly, by $w(x, y, \alpha)=c$. In this expression the $y$ indicates the presence of the $n$ coördinates and $\alpha$ that of the $n$ constants.

* Bolza, Vorlesungen, p. 106. 
The derivatives of the transversal function are known to be

$$
w_{x}=f(x, y, p)-\sum p_{i} f_{p i}, \quad w_{y i}=f_{p i} \quad(i=1,2, \cdots, n) .
$$

These functions are formed by replacing the derivatives in the integrand function by the slope functions of the field. Along an extremal we have, of course,

$$
\frac{d y_{i}}{d x}=p_{i}(x, y, \alpha) .
$$

The $n$ equations which define the extremals may be derived from the transversal function by differentiation with respect to the constants.* They are

$$
w_{\alpha}=(x, y, \alpha)=\gamma \quad(i=1,2, \cdots, n) .
$$

The slope functions of the field can be obtained by differentiating these equations with respect to $x$.

We obtain the $n$ equations

$$
w_{\alpha_{i} x}+\sum p_{j} w_{\alpha_{i} y_{j}}=0 \quad(i=1,2, \cdots, n) .
$$

These can be solved for the $p_{i}$ since we are not concerned with regions for which the determinant of this system vanishes. In fact, one of the restrictions introduced in the direct problem is that the derivatives must remain finite.

2. A transversality for $n$ dependent variables will be defined as a set of $n$ relations

$$
w_{y_{i}}=m_{i} w_{x}
$$

which satisfy the inequality

$$
1+\sum p_{i} m_{i} \not \equiv 0
$$

and the $n(n-1) / 2$ equations

$$
\sum p_{k}\left(m_{k} \frac{\partial m_{i}}{\partial p_{i}}-m_{i} \frac{\partial m_{k}}{\partial p_{i}}\right)+\frac{\partial m_{i}}{\partial p_{j}}=\sum p_{k}\left(m_{k} \frac{\partial m_{i}}{\partial p_{i}}-m_{j} \frac{\partial m_{k}}{\partial p_{i}}\right)+\frac{\partial m_{i}}{\partial p_{i}}
$$

By substituting in these conditions the derivatives of the transversal function we shall show that these conditions are always satisfied in any problem.

From (1) and (4) it follows that

$$
m_{i}=\frac{f_{p_{i}}}{f-\sum f_{p_{j}} p_{i}} .
$$

\footnotetext{
* Bolza, Vorlesungen, p. 601.
} 
If the inequality (5a) were not satisfied we would have

$$
1+\frac{\sum f_{p_{i}} p_{i}}{f-\sum f_{p_{j}} p_{i}} \equiv 0,
$$

and, therefore, $f(x, y, p) \equiv 0$.

To show that the $n(n-1) / 2$ equations (5b) are always satisfied we substitute in them the derivatives of the transversal function. In order to introduce logarithms we first divide by $m_{i} m_{j}$. The left member of $(5 b)$ then becomes

$$
\begin{aligned}
& \frac{1}{m_{j}} {\left[\sum p_{k} m_{k} \frac{\partial}{\partial p_{j}} \log \frac{m_{i}}{m_{k}}+\frac{\partial}{\partial p_{j}} \log m_{i}\right] } \\
&= \frac{f-\sum f_{p_{k} p_{k}}}{f_{p_{j}}}\left[\frac{\sum p_{k} f_{p_{k}}}{f-\sum f_{p_{k} p_{k}}}\left(\frac{f_{p_{i} p_{j}}}{f_{p_{i}}}-\frac{f_{p_{k} p_{j}}}{f_{p_{k}}}\right)+\frac{f_{p_{i} p_{j}}}{f_{p_{i}}}\right. \\
&\left.\quad-\frac{f_{p_{j}}-f_{p_{j}}-\sum f_{p_{k} p_{j} p_{k}}}{f-\sum f_{p_{k}} p_{k}}\right] \\
&=\frac{1}{f_{p_{j}}}\left[\frac{f_{p_{i} p_{j}}}{f_{p_{i}}} \sum f_{p_{k} p_{k}}-\sum f_{p_{k} p_{j} p_{k}}\right. \\
&\left.\quad+\frac{f_{p_{i} p_{j}}}{f_{p_{i}}}\left(f-\sum f_{p_{k} p_{k}}\right)+\sum f_{p_{k} p_{j} p_{k}}\right] .
\end{aligned}
$$

In this last expression the summations cancel and we have, finally,

$$
f f_{p_{i} p_{j}} / f_{p_{i}} f_{p_{j}} \cdot
$$

Since this is symmetrical in the derivatives with respect to $p_{i}$ and $p_{j}$ it follows that any transversality which arises in a problem necessarily satisfies equations $(5 \mathrm{~b}) .^{*}$

* It is significant that if relations (5a) and (5b) hold for any set of variables, $y_{i}$, they also hold for any other set, $z_{i}=Z_{i}(y)$. Employing the tensor notation for the moment we see that

$$
p^{i}=\frac{\partial y_{i}}{\partial z_{k}} q^{k}, \quad m_{i}=\frac{\partial z_{i}}{\partial y_{i}} n_{i},
$$

where $q^{k}=d z_{k} / d x$ and $n_{i}=\left(\partial w / \partial z_{j}\right) /(\partial w / \partial x)$ are the components of the tensor in the new variables. By means of these expressions we see that $(5 a)$ becomes

Hence this quantity is an invariant.

$$
1+p^{i} m_{i}=1+\frac{\partial y_{i}}{\partial z_{k}} \frac{\partial z_{j}}{\partial y_{i}} q^{k} n_{j}=1+q^{i} n_{j} .
$$

The equations (5b) are tensor equations formed from the components of a covariant tensor of rank 2. In addition to the relations already mentioned we have

$$
R_{i j}=S_{r i} \frac{\partial z_{r}}{\partial y_{i}} \frac{\partial z_{i}}{\partial y_{i}}
$$


3. We now differentiate equations (4) with respect to one of the constants and obtain

$$
\frac{1}{w_{x}} \frac{\partial w_{y_{i}}}{\partial \alpha_{j}}-\frac{w_{y_{i}}}{w_{x}^{2}} \frac{\partial w_{x}}{\partial \alpha_{j}}=\frac{\partial m}{\partial \alpha_{j}} \quad(i=1,2, \cdots, n) .
$$

On replacing $w_{y_{i}}$ by $m_{i} w_{x}$, according to (4), these become

$$
\frac{1}{w_{x}} \frac{\partial w_{y_{i}}}{\partial \alpha_{i}}-\frac{m_{i}}{w_{x}} \frac{\partial w_{x}}{\partial \alpha_{j}}=\frac{\partial m_{i}}{\partial \alpha_{j}} .
$$

Now we multiply each of these $n$ equations by $-p_{i}$ and add, with the result

$$
-\frac{1}{w_{x}} \sum p_{i} \frac{\partial w_{y_{i}}}{\partial \alpha_{j}}+\frac{1}{w_{x}} \frac{\partial w_{x}}{\partial \alpha_{j}} \sum m_{i} p_{i}=-\sum p_{i} \frac{\partial m_{i}}{\partial \alpha_{i}} .
$$

Substituting $\partial w_{x} / \partial \alpha_{i}$ for the first summation in the left member, by (3), the last equation reduces to

$$
\frac{1}{w_{x}}\left(1+\sum m_{i} p_{i}\right) \frac{\partial w_{x}}{\partial \alpha_{j}}=-\sum p_{i} \frac{\partial m_{i}}{\partial \alpha_{j}} .
$$

Now by adding and subtracting the term $\sum m_{i}\left(\partial p_{i} / \partial \alpha_{j}\right)$ in the right member, and then dividing by $1+\sum m_{i} p_{i}$, we finally obtain

(6) $\quad \frac{1}{w_{x}} \frac{\partial w_{x}}{\partial \alpha_{i}}=-\frac{\partial}{\partial \alpha_{j}} \log \left(1+\sum m_{i} p_{i}\right)+\frac{1}{1+\sum m_{i} p_{i}}\left(\sum m_{i} \frac{\partial p_{i}}{\partial \alpha_{j}}\right)$.

If, as in the case of two variables, $\delta$ represents the change in a function when the $\alpha_{i}$ are varied we may write

$$
\delta \log w_{x}=\sum \frac{1}{w_{x}} \frac{\partial w_{x}}{\partial \alpha_{j}} \delta \alpha_{j}
$$

Forming the right member of this equation by means of (6) we find

$$
\delta \log w_{x}=-\delta \log \left(1+\sum m_{i} p_{i}\right)+\frac{\sum m_{i} \delta p_{i}}{1+\sum m_{i} p_{i}} .
$$

The first term on the right is exact. The second is exact if

$$
\frac{\partial H_{p_{j}}}{\partial p_{i}}=\frac{\partial H_{p_{i}}}{\partial p_{j}}
$$

where $R_{i j}=\partial m_{i} / \partial p_{i}$ and $S_{r i}=\partial n_{r} / \partial q_{v e}$ Upon substituting these expressions in the left members of $(5 \mathrm{~b})$ the result is, after some reduction,

$$
p^{k}\left(m_{k} R_{i j}-m_{i} R_{k j}\right)+R_{i j}=\left[q^{r}\left(n_{r} S_{a b}-n_{a} S_{r b}\right)+S_{a b}\right] \frac{\partial z_{a}}{\partial y_{i}} \frac{\partial z_{b}}{\partial y_{i}}
$$

which displays the tensorial nature of the equations. If $T_{i j}$ represents the left member of $(5 \mathrm{~b})$ the equations themselves may be written as $T_{i j}-T_{i i}=0$. 
where $H p_{i}$ is the coefficient of $\delta p_{i}$. Now this condition reduces to

$\sum p_{k}\left(m_{k} \frac{\partial m_{i}}{\partial p_{i}}-m_{i} \frac{\partial m_{k}}{\partial p_{j}}\right)+\frac{\partial m_{i}}{\partial p_{i}}-m_{i} m_{j}=\sum p_{k}\left(m_{k} \frac{\partial m_{j}}{\partial p_{i}}-m_{j} \frac{\partial m_{k}}{\partial p_{i}}\right)+\frac{\partial m_{j}}{\partial p_{j}}-m_{j}, m_{i}$.

But this is the same as (5b) when we omit the identical term $-m_{i} m_{j}$. Since we have supposed (5b) to be satisfied, the right member of (7) is always exact.

On integration we have

$$
w_{x}=\frac{g(x, y) e^{\Theta}}{1+\sum m_{i} p_{i}}
$$

where $g$ contains $x$ and the dependent variables $y_{i}$ but is independent of the $\alpha_{i}$, and

$$
\Theta=\int \frac{\sum m_{i} \delta p_{i}}{1+\sum m_{i} p_{i}}
$$

From equations (1) we can eliminate the partial derivatives, $f_{p_{i}}$, and find

$$
f(x, y, p)=w_{x}+\sum w_{y_{i}} p_{i} .
$$

On account of (4) we have

$$
w_{y_{i}}=\frac{m_{i} g(x, y) e^{\Theta}}{1+\sum m_{i} p_{i}} .
$$

Eliminating the derivatives of the transversal function by these last equations we have, finally,

$$
f(x, y, p)=g(x, y) e^{\ominus} .
$$

4. We have now to show how the function $g(x, y)$ is to be determined when a set of extremals is prescribed in addition to the transversality. Let us suppose, then, that in addition to the transversality a family of extremals involving $2 n$ parameters is prescribed, that they have been solved for any $n$ of these parameters, and are given by

$$
\psi_{1}\left(x, y_{1}, y_{2}, \cdots, y_{n}, a_{1}, a_{2}, \cdots, a_{n}\right)=\psi_{i}(x, y, a)=c_{i} .
$$

Since they are to define the extremals we shall suppose that the functions $\psi_{i}$ are of class $C^{\prime}$.

If for a certain set of values of the coördinates the determinant

$$
D(\psi)=\frac{\partial\left(\psi_{1}, \psi_{2}, \cdots, \psi_{n}\right)}{\partial\left(y_{1}, y_{2}, \cdots, y_{n}\right)}
$$

does not vanish we can solve for the $y_{i}$ in terms of $x$ and the constants. We may then substitute these expressions in (11) and write the identities

$$
\psi_{i}(x, y(x, a, c), a)=c_{i} \quad(i=1,2, \cdots, n) .
$$


We can differentiate these identities with respect to $c_{k}$, finding

and

$$
\sum \frac{\partial \psi_{i}}{\partial y_{j}} \frac{\partial y_{j}}{\partial c_{k}}=0 \quad(i=1,2, \cdots, n, \text { except } k),
$$

$$
\sum \frac{\partial \psi_{k}}{\partial y_{j}} \frac{\partial y_{j}}{\partial c_{k}}=1
$$

Upon solving these equations for $\partial y_{h} / \partial c_{k}$ we obtain

$$
\frac{\partial y_{h}}{\partial c_{k}}=\frac{C(k, h)}{D(\psi)},
$$

where $C(k, h)$ is the cofactor of $\partial \psi_{k} / \partial y_{h}$ in $D(\psi)$.

We now form the determinant of the derivatives $\partial y_{h} / \partial c_{k}$ and find

$$
\frac{\partial\left(y_{1}, y_{2}, \cdots, y_{n}\right)}{\partial\left(c_{1}, c_{2}, \cdots, c_{n}\right)}=\frac{1}{D(\psi)}
$$

by the theorem on reciprocal determinants.* Since $D(\psi)$ is finite on account of the continuity of the functions this determinant is different from zero. Moreover, it is finite in the region for which $D(\psi) \not \equiv 0$. This is a necessary and sufficient condition for the existence of a field of extremals. $\dagger$

The existence of the field implies the existence of an invariant integral or transversal function containing $n$ constants, $\alpha_{i}$; as parameters. If this transversal function were known the extremals could be obtained as usual by differentiation. They would be given by

$$
\frac{\partial w(x, y, \alpha)}{\partial \alpha_{i}}=\gamma_{i} \quad(i=1,2, \cdots, n) .
$$

The extremals given by equations (13) are the same as those given by (11). It follows that the slope functions of the field, $\pi_{i}$, as defined by

$$
\frac{\partial w_{x}}{\partial \alpha_{i}}+\sum \pi_{j} \frac{\partial w_{y_{j}}}{\partial \alpha_{i}}=0 \quad(i=1,2, \cdots, n)
$$

are identical with those defined by

$$
\frac{\partial \psi_{i}}{\partial x}+\sum p_{i} \frac{\partial \psi_{i}}{\partial y_{i}}=0 \quad(i=1,2, \cdots, n) .
$$

* Weld, Theory of Determinants, p. 139.

† Bolza, Vorlesungen, p. 635. 
In a field the determinants of these systems are different from zero. If they were not, the slope functions would become infinite, which is not the case when a field exists.

Since $p_{i}(x, y, a)=\pi_{i}(x, y, \alpha)$ it follows that there must exist transformations such as

$$
a_{i}=A_{i}(\alpha), \quad \alpha_{i}=A_{i}^{\prime}(a) \quad(i=1,2, \cdots, n) .
$$

This is a consequence of the fact that the $n$ constants $a_{i}$ are distinct and likewise the $n$ constants $\alpha_{i}$.

We shall now show that no one of the functions*

$$
m_{i}(x, y, \pi(x, y, \alpha))
$$

can be independent of all the constants. For if it were we would have

$$
\frac{\partial m_{k}}{\partial \alpha_{j}}=\frac{1}{w_{x}^{2}}\left[\frac{\partial^{2} w}{\partial \alpha_{j} \partial y_{k}} \frac{\partial w}{\partial x}-\frac{\partial w}{\partial y_{k}} \frac{\partial^{2} w}{\partial \alpha_{j} \partial x}\right]=0 \quad(j=1,2, \cdots, n),
$$

and consequently

$$
\frac{\partial^{2} w}{\partial \alpha_{j} \partial x}=\frac{\partial^{2} w}{\partial \alpha_{j} \partial y_{k}} \frac{\partial w}{\partial x} \frac{1}{\partial w / \partial y_{k}}=\frac{1}{m_{k}} \frac{\partial^{2} w}{\partial \alpha_{j} \partial y_{k}} .
$$

If we substitute this result in equations (14) they become

$$
\left(\pi_{k}+\frac{1}{m_{k}}\right) \frac{\partial^{2} w}{\partial \alpha_{j} \partial y_{k}}+{ }_{k} \sum \pi_{i} \frac{\partial^{2} w}{\partial \alpha_{j} \partial y_{i}}=0 \quad(j=1,2, \cdots, n) .
$$

The subscript $k$ at the left of the summation denotes the omission of that term.

Since we have limited ourselves to a region in which $1+\sum m_{i} \pi_{i} \neq 0$ the functions $\pi_{i}$ and $\pi_{k}+1 / m_{k}$ cannot all be identically zero. Therefore the determinant of the coefficients of the preceding equations must vanish. But this determinant is the same as that of (14) which does not vanish. From this contradiction it follows that $m_{k}$ is not independent of all the parameters.

If $m_{k}$ were expressed in terms of $p_{i}(x, y, a)$ and were independent of all the parameters $a_{i}$ we would then have

* In this connection it should be pointed out that the problem degenerates if any one of the functions $m_{i}$ is independent of all the slope functions, $p_{i}$. If this were the case we would have $w_{y_{i}}=$ $m_{i} w_{x}$ where $m_{i}$ is a function of the coördinates only and does not contain the canonical constants of the problem. If a change of variables is made so that the new independent variable, $\xi$, satisfies the equation, $\xi_{y_{i}}-m_{i} \xi_{x}=0$, then it is possible to choose the other new variables, $\eta$, so as to make one of the derivatives, $w_{\eta_{i}}$, identically zero. This means of course that the derivative $d \eta_{i} / d \xi$ is absent from the integrand function when expressed in terms of the new variables. 


$$
\frac{\partial m_{k}}{\partial a_{j}}=\sum \frac{\partial m_{k}}{\partial a_{i}} \frac{\partial \alpha_{i}}{\partial a_{j}} \equiv 0 \quad(j=1,2, \cdots, n) .
$$

Since the derivatives $\partial m_{k} / \partial \alpha_{i}$ cannot all be zero the determinant is zero; that is,

$$
\frac{\partial\left(\alpha_{1}, \alpha_{2}, \cdots, \alpha_{n}\right)}{\partial\left(a_{1}, a_{2}, \cdots, a_{n}\right)} \equiv 0 .
$$

But if this were true we could not obtain the transformations (16). We conclude, therefore, that $m_{k}$ contains at least one of the constants $a_{i}$.

We have found in (8) that

$$
w_{x}=\frac{g(x, y) e^{\Theta}}{1+\sum m_{i} p_{i}}
$$

and in (8a)

$$
w_{y_{i}}=\frac{m_{i} g(x, y) e^{\ominus}}{1+\sum m_{i} p_{i}} .
$$

Since $w$ is of class $C^{\prime \prime}$ the derivatives $w_{x y_{i}}$ and $w_{y_{i} x}$ are equal. We express this fact by the $n$ relations

$$
\frac{\partial}{\partial y_{k}}\left(\frac{g e^{\Theta_{i}}}{1+\sum m_{i} p_{i}}\right)=\frac{\partial}{\partial x}\left(\frac{m_{k} g e^{\Theta}}{1+\sum m_{i} p_{i}}\right) .
$$

These reduce to

$$
\begin{aligned}
m_{k} \frac{g_{z}}{g}-\frac{g_{y_{k}}}{g} & =\left[\frac{\partial \theta}{\partial y_{k}}-\frac{\partial}{\partial y_{k}} \log \left(1+\sum m_{i} p_{i}\right)\right. \\
& \left.=\frac{\partial m_{k}}{\partial x}-m_{k}\left(\frac{\partial \theta}{\partial x}-\frac{\partial}{\partial x} \log \left(1+\sum m_{i} p_{i}\right)\right)\right] .
\end{aligned}
$$

Solutions of the $n$ equations (17) can be obtained as before by using the fact that $g$ and its derivatives are independent of the constants $a_{i}$ as well as the canonical constants $\alpha_{i}$.

If we designate the right members of (17) by $\Phi_{k}$, then the derivatives of $\log g$ are given by

$$
\begin{gathered}
\frac{g_{x}}{g}=\frac{1}{\partial m_{k} / \partial a_{i}} \frac{\partial}{\partial a_{i}} \Phi_{k}, \\
\frac{g_{y_{k}}}{g}=\frac{m_{k}}{\partial m_{k} / \partial a_{i}} \frac{\partial}{\partial a_{i}} \Phi_{k}-\Phi_{k} \quad(i, k=1,2,3, \cdots, n) .
\end{gathered}
$$

From these $g$ can be found by quadrature. 
The conditions to be satisfied by a $2 n$-parameter family of curves in a space of $n+1$ dimensions if it is to represent the extremals of a problem with a prescribed transversality are at once apparent. The $n$ expressions (18) for $g_{x} / g$ must be identical and, likewise, the expressions (19) for $(1 / g) \partial g / \partial y_{k}$. Moreover, these must actually be independent of the constants, that is,

$$
\frac{\partial}{\partial \alpha_{i}}\left(\frac{1}{\partial m_{k} / \partial a_{j}} \frac{\partial}{\partial a_{j}} \Phi_{k}\right) \equiv 0 \quad(i, j=1,2, \cdots, n) .
$$

Also, if the expressions (18) and (19) are actually to represent a function we must have

$$
\begin{aligned}
& \frac{\partial^{2} \log g}{\partial y_{k} \partial x}=\frac{\partial^{2} \log g}{\partial x \partial y_{k}}, \\
& \frac{\partial^{2} \log g}{\partial y_{k} \partial y_{h}}=\frac{\partial^{2} \log g}{\partial y_{h} \partial y_{k}} .
\end{aligned}
$$

Finally, the slope functions of the given curves must satisfy $w_{y_{h} y_{k}}=w_{y} y_{h}$, or

$$
\frac{\partial}{\partial y_{k}}\left(\frac{m_{k} g e^{\Theta}}{1+\sum m_{i} p_{i}}\right)=\frac{\partial}{\partial y_{k}}\left(\frac{m_{k} g e^{\Theta}}{1-\sum m_{i} p_{i}}\right) .
$$

These conditions might have been imposed along with (17). They were omitted at that time because, as we shall now show, they are satisfied by the same function, $g$, if they are satisfied at all.

Let us suppose that the equations (17) are satisfied by $g\left(x, y_{1}, y_{2}, \cdots, y_{n}\right)$. If we multiply the $k$ th and the $h$ th equations by $m_{h}$ and $-m_{k}$, respectively, and add, the result is

$$
\begin{aligned}
& m_{h} \frac{\partial}{\partial y_{k}}\left(\frac{g e^{\Theta}}{1+\sum m_{i} p_{i}}\right)-m_{k} \frac{\partial}{\partial y_{h}}\left(\frac{g e^{\Theta}}{1+\sum m_{i} p_{i}}\right) \\
& =\frac{\partial e^{\Theta}}{1+\sum m_{i} p_{i}}\left(m_{h} \frac{\partial m_{k}}{\partial x}-m_{k} \frac{\partial m_{h}}{\partial x}\right)=\frac{g e^{\Theta}}{1+\sum m_{i} p_{i}}\left(\frac{w_{y h}}{w_{x}^{2}} w_{y_{k} x}-\frac{w_{y_{k}}}{w_{x}^{2}} w_{y_{h}^{x}}\right) .
\end{aligned}
$$

Now we make use of the fact that $w_{y_{k} y_{h}}-w_{y_{h} y_{k}}=0$, by adding to the right member of the previous equation

That equation then becomes

$$
\frac{g e^{\Theta}\left(w_{y_{k} y_{h}}-w_{y_{h} y_{k}}\right)}{1+\sum m_{i} p_{i} w_{x}}
$$

$$
\begin{gathered}
m_{h} \frac{\partial}{\partial y_{k}}\left(\frac{g e^{\Theta}}{1+\sum m_{i} p_{i}}\right)-m_{k} \frac{\partial}{\partial y_{h}}\left(\frac{g e^{\Theta}}{1+\sum m_{i} p_{i}}\right)=\left(\frac{g e^{\Theta}}{1+\sum m_{i} p_{i}}\right)\left[\frac{\partial}{\partial y_{h}}\left(\frac{w_{y_{k}}}{w_{x}}\right)\right. \\
\left.-\frac{\partial}{\partial y_{k}}\left(\frac{w_{y_{h}}}{w_{x}}\right)\right]=\frac{g e^{\Theta}}{1+\sum m_{i} p_{i}}\left(\frac{\partial}{\partial y_{h}}-m_{k} \frac{\partial m_{h}}{\partial y_{k}}\right) .
\end{gathered}
$$


This final form is identical with $\left(17^{\prime}\right)$ and shows that these equations are satisfied by the same function, $g\left(x, y_{1}, y_{2}, \cdots, y_{n}\right)$, that satisfies (17).

5. We shall conclude this section by showing directly that if the conditions which we have enumerated are satisfied and an integrand function, $f=g e^{\theta}$, has been determined, then the given curves satisfy the Euler equations and are the extremals of the problem.

We know that the slope functions of a particular sub-family of the given curves satisfy*

$$
\begin{aligned}
& \frac{\partial}{\partial y_{i}}\left[f-\sum p_{k} f_{p_{k}}\right]=\frac{\partial}{\partial x}\left[f_{p_{i}}\right], \\
& \frac{\partial}{\partial y_{i}}\left[f_{p_{k}}=\frac{\partial}{\partial y_{k}}\left[f_{p_{i}}\right] .\right.
\end{aligned}
$$

When we carry out the differentiation (17) becomes

$$
\left.f_{y_{i}}+\sum f_{p_{k} p_{k y_{i}}}-\sum p_{k y_{i}} f_{p_{k}}-\sum p_{k} \frac{\partial}{\partial y_{i}}\left[f_{p_{k}}\right]-f_{p_{i} x}-\sum\right]_{p_{i} p_{k} p_{k x}}=0 \text {. }
$$

Then, by omitting terms which cancel and making use of $\left(17^{\prime}\right)$ we find

$$
f_{y_{i}}-f_{p_{i} x}-\sum \frac{\partial}{\partial y_{k}}\left[f_{p_{i}}\right] p_{k}-\sum f_{p_{i} p_{k} p_{k x}}=0
$$

which may be written

$$
f_{y_{i}}-\frac{d}{d x} f_{p_{i}}=0 \quad(i=1,2, \cdots, n)
$$

Since these are the Euler equations of the problem the given curves are actually the extremals of the integrand function which we have determined by means of them.

Example IV. Let us suppose that the set of functions which determine the transversality are

$$
m_{i}=\frac{2 p_{i}}{x^{2}-\sum p_{k}^{2}} \quad(i=1,2, \cdots, n) .
$$

First, we show that this set satisfies equations (5b) and is really a transversality. The left member of that equation becomes

$$
\sum p_{k}\left[\frac{2 p_{k}}{x^{2}-\sum p_{k}^{2}} \frac{4 p_{i} p_{j}}{\left(x^{2}-\sum p_{k}^{2}\right)^{2}}-\frac{2 p_{i}}{\left(x^{2}-\sum p_{k}^{2}\right)} \frac{4 p_{i} p_{k}}{\left(x^{2}-\sum p_{k}^{2}\right)^{2}}\right]+\frac{4 p_{i} p_{i}}{x^{2}-\sum p_{k}^{2}} .
$$

* In this paragraph the use of the square brackets, [ ], indicates that the differentiation is to be carried out after $p$ has been replaced by $p\left(x, y_{1}, y_{2}, \cdots, y_{n}, \alpha_{1}, \alpha_{2}, \cdots, \alpha_{n}\right)$. 
The terms under the summation sign are all zero and the last term is symmetrical in $p_{i} p_{j}$. Since substitution in the right member would give the same result the condition is satisfied.

Now we may calculate $\Theta$ by means of (9) and find

$$
\Theta=\log \left(x^{2}+\sum p_{k}^{2}\right) .
$$

Equation (8) then gives us the integrand function, which is

$$
f=g(x, y)\left(x^{2}+\sum p_{k}^{2}\right) .
$$

We shall now assume, further, that the extremals are given by the equations

$$
y_{i}=\left(a_{i} x^{3}-c_{i}\right) / 3 \quad(i=1,2, \cdots, n) .
$$

We can form a field by giving fixed values to the $a_{i}$. In this case the slope functions are

$$
p_{i}=a_{i} x^{2} .
$$

Then we calculate $\Phi_{k}$ by means of (17). This gives, if we denote $\sum a_{i}{ }^{2}$ by $A^{2}$,

$$
\begin{aligned}
\Phi_{k} & =-\frac{4 a_{k} x A^{2}}{1-x^{2} A^{2}}-\frac{2 a_{k}}{1-x^{2} A^{2}}\left(\frac{2}{x}+\frac{2 x A^{2}}{1+x^{2} A^{2}}-\frac{2 x A}{1+x^{2} A^{2}}-\frac{2 x A^{2}}{1-x^{2} A^{2}}\right) \\
& =-\frac{2 a_{k}}{1-x^{2} A^{2}} \frac{2}{x}=-m_{k} \frac{2}{x} .
\end{aligned}
$$

Substituting these results in (18) and (19) we find

$$
\frac{g_{x}}{g}=-\frac{2}{x}, \text { and } \frac{g_{y_{k}}}{g}=0 .
$$

Omitting the constant of integration we have, therefore,

and

$$
g=1 / x^{2},
$$

$$
f=(1 / x)^{2}\left(x^{2}+\sum p_{?}^{2}\right) .
$$

The conditions which we have found are all satisfied and the given curves are actually the extremals of the integrand function.*

\footnotetext{
* Since this paper was prepared, Douglas has published a paper in these Transactions (April, 1927), entitled Extremals and transversality of the general calculus of variations problem of the first order in space. The subject matter of his paper has a good deal in common with that of the present paper, but the problem is given a geometric rather than an analytic interpretation.
}

YALE UNIVERSITY, New HAVEN, CoNn. 